\title{
Barriers to lifestyle changes for prevention of cardiovascular disease - a survey among 40-60-year old Danes
}

\author{
Jesper Bo Nielsen ${ }^{1 *}$, Anja Leppin², Dort e Gyrd-Hansen³ ${ }^{3}$ Dorte Ejg Jarbøl ${ }^{1}$, Jens Søndergaard ${ }^{1}$ \\ and Pia Veldt Larsen ${ }^{4}$
}

\begin{abstract}
Background: Elimination of modifiable risk factors including unhealthy lifestyle has the potential for prevention of $80 \%$ of cardiovascular disease cases. The present study focuses on disclosing barriers for maintaining specific lifestyle changes by exploring associations between perceiving these barriers and various sociodemographic and health-related characteristics.

Methods: Data were collected through a web-based questionnaire survey and included 962 respondents who initially accepted treatment for a hypothetical cardiovascular risk, and who subsequently stated that they preferred lifestyle changes to medication. Logistic regression was used to analyse associations between barriers to lifestyle changes and relevant covariates.

Results: A total of $45 \%$ of respondents were identified with at least one barrier to introducing 30 min extra exercise daily, $30 \%$ of respondents reported at least one barrier to dietary change, and among smokers at least one barrier to smoking cessation was reported by $62 \%$ of the respondents. The perception of specific barriers to lifestyle change depended on sociodemographic and health-related characteristics.

Conclusion: We observed a considerable heterogeneity between different social groups in the population regarding a number of barriers to lifestyle change. Our study demonstrates that social inequality exists in the ability to take appropriate preventive measures through lifestyle changes to stay healthy. This finding underlines the challenge of social inequality even in populations with equal and cost-free access to health care. Our study suggests supplementing traditional public campaigns to counter cardiovascular disease by using individualized and targeted initiatives.
\end{abstract}

Keywords: Cardiovascular disease, Prevention, Survey, Lifestyle, Barriers

\section{Background}

The prevalence of cardiovascular mortality in Denmark and comparable countries has decreased during recent decades, but cardiovascular disease (CVD) remains a dominant cause of morbidity and mortality in many countries $[1,2]$. Elimination of modifiable risk factors including unhealthy lifestyle allows for prevention of $80 \%$ of CVD cases [3], and individuals with desirable lifestyle factors (not smoking, physically active, healthy diet, BMI $<25)$ are expected to have a $67-72 \%$ lower risk of

\footnotetext{
* Correspondence: jbnielsen@health.sdu.dk

${ }^{1}$ Research Unit for General Practice, Department of Public Health, University

of Southern Denmark, J.B.Winsløwvej 9, DK-5000 Odense, Denmark

Full list of author information is available at the end of the article
}

developing heart failure [4], However, actual implementation and maintenance of preventive measures, e.g. lifestyle change, has proven difficult [5-7]. Experiencing barriers to lifestyle change has been shown to prevent successful change of low exercise levels, unhealthy nutrition and/or smoking status among at-risk groups as well as in general populations [8-13].

Inability to maintain changes may be explained by limited structural resources (e.g. available time, or lacking financial resources, and influence by partner) or more personal challenges (e.g. habits, taste, and previous experience) [14]. Which types of barriers one perceives may, however, differ across population groups. In particular socio-economic factors might make a difference, 
but few studies have as yet systematically compared barrier perceptions across socio-economic strata. If specific barriers to lifestyle change can be identified and linked to specific population groups, it may be beneficial to approach lifestyle changes through targeted or individually tailored messages boosting self-efficacy and coping abilities, rather than using traditional and more generalized risk messages addressing mainly negative consequences of given lifestyles.

A recent survey of 40-60 year old Danes demonstrated that the vast majority of the study population expressed a preference for lifestyle changes instead of lifelong medication to prevent a hypothetical heart disease [15]. At the same time the respondents expressed doubt that they would be able to maintain the changed lifestyle for an extended period of time [16]. The present article is based on the same study population but focuses on disclosing group differences in perceived difficulties for maintaining specific lifestyle changes including exercise, diet, and smoking. The study explores whether perception of barriers commonly identified in the literature differs between socio-demographic groups and groups with different health status, different weight and different levels of physical activity.

In particular, the present study aims at investigating whether middle-aged Danes from different socioeconomic backgrounds and with different health status vary in their perception of

1) Inexperience, time and cost as barriers to engaging in exercise

2) Taste, time and cost as barriers to eating low-fat food

3) Previous unsuccessful experience or lack of support from co-habitant(s) as barriers to stopping smoking

\section{Methods}

\section{Sample and setting}

A representative sample of 40-60 year old persons was invited to participate. This age group was considered to represent the most likely first time users of preventive therapy against CVD. The respondents were presented with a hypothetical scenario asking them to imagine that they were at increased risk of heart disease. Subsequently they were offered a hypothetical preventive medical intervention targeted at reducing the risk of heart disease. No medication name was mentioned. Afterwards, subjects were provided with the choice to either accept or reject this medication and further to indicate their preference for lifestyle changes versus medication. Lifestyle changes were framed as having the same benefit on CVD risk as medication. Eventually, respondents were asked about perceived barriers to maintaining specified lifestyle changes for 1 year (Table 1).
Table 1 Question presented to all respondents who had accepted treatment in the first place, but preferred lifestyle changes (another $1 / 2$ hour daily exercise + low fat diet + no smoking) to medication

Which (lifestyle change) do you think will be hardest to maintain? (multiple answers are allowed)

- Daily exercise for another $1 / 2$ hour - I am not used to doing physical exercise

- Daily exercise for another $1 / 2$ hour - Work and children makes it hard to find the time

- Daily exercise for another $1 / 2$ hour - It is expensive to go to a fitness center

- Low fat diet - It takes longer to cook compared to the usual cooking - Low fat diet - I don't like low fat food

- Low fat diet - many vegetables and fish are more expensive than the usual food

o Stop smoking - I have tried several times

o Stop smoking - I don't think I can stop smoking when my cohabitant continues smoking

○ Other, please specify

Data were collected through a web-based questionnaire in March 2012 using TNS Gallup's web-panel GallupForum (a panel consisting of 40.000+ Danish speaking persons $>14$ years of age). A random sample of 3928 panel members aged 40-60 years received an invitation by e-mail to visit a website, $2346(60 \%)$ accessed the website, and 2099 (91\%) answered the questionnaire. The full sample (18 groups) included variations over the size of risk reduction as well as delay in time before treatment benefit. The present paper uses a sub-sample (9 groups) consisting of those respondents who were presented with an immediate treatment benefit as an absolute risk reduction of $10 \%$ to $5 \%$ ( $n=1069$ out of 2099), who subsequently stated that they preferred lifestyle changes $(1 / 2$ hour daily exercise + low fat diet + no smoking) to medication ( $n=962$ out of 1069).

According to the Act on a Biomedical Research Ethics Committee System in Denmark, the project was not a biomedical research project and therefore did not need the ethic committee's approval. All respondents were anonymous.

\section{Questionnaire}

The questionnaire described a risk scenario together with 12 questions concerning health, such as self-rated health status, experience with heart disease (own/family), smoking, physical activity and willingness to accept treatment under various conditions, plus a number of questions on sociodemographic characteristics (gender, age, highest educational attainment, household income). The questionnaire has been used in previous studies $[15,16]$ except for questions on barriers. Based on the reviewed literature, three types of lifestyle change were selected together with two-three specific potential barriers to maintaining each of these changes for a year. The respondents were asked to state whether each of the barriers applied to them (Table 1). In addition, a free text option was provided to report further barriers. Prior to being 
presented to the web-panel, the entire questionnaire was evaluated regarding comprehensibility, relevance, acceptability and feasibility, and pilot tested by TNS Gallup. All respondents received a lottery ticket with the chance of winning goods worth 50 USD.

\section{Statistical analyses}

Univariate and multivariable logistic regression was used to analyse associations between each predefined barrier to lifestyle changes and a number of covariates (gender, age, self-rated health status, body mass index (BMI), physical activity, experience with heart disease, education, work status, and household income). Tests for trends were conducted when appropriate, i.e. for continuous and ordinal variables. Only results from the multiple analyses, adjusted for gender and age as potential confounders, are presented in the paper. All analyses were conducted using Stata Release 11 (StataCorp, College Station, TX, USA).

\section{Results}

Socio-economic characteristics of the 962 respondents who accepted the intervention and preferred life style changes are listed in Table 2 . This subsample did not differ from the entire group of respondents $(n=2099)$ based on gender, age distribution, health status, household income, educational attainment, or occupational status. Moreover, for the age strata used, our sample of respondents was representative of the Danish population in terms of household income, educational attainment, and occupational status (data not shown).

\section{Barrier to life style change: increased daily exercise for $30 \mathrm{~min}$}

A total of $45 \%$ of respondents indicated at least one of the three predefined barriers to introducing $30 \mathrm{~min}$ extra exercise daily. Lack of time for exercise was the most frequently reported barrier followed by not being used to do exercise and the cost of participating in organized exercise activities (Fig. 1).

The younger the respondents and the higher the income and level of educational attainment, the more often lack of time was reported as a barrier to $1 / 2$ hour extra daily exercise (Table 3 ). Respondents in the work force significantly more often saw time as a barrier to exercise than those not working ( $\mathrm{OR}=5.70, p<0.001)$.

Respondents already reporting high levels of physical activity less often saw lack of time for another 30 min of daily exercise as a barrier to maintaining a changed lifestyle $(\mathrm{OR}=0.52, \mathrm{p}<0.001)$. We did not observe a significant association between lack of time for exercise and body mass index (BMI).

The lower the income the more frequently respondents perceived not being accustomed to exercising as a barrier.
Table 2 Sample characteristics

\begin{tabular}{|c|c|}
\hline Total, n & $\begin{array}{l}\text { Participants } \\
962\end{array}$ \\
\hline \multicolumn{2}{|l|}{ Gender, n (\%) } \\
\hline female & $480(49.9)$ \\
\hline male & $482(50.1)$ \\
\hline Age, mean (years) & 50.6 \\
\hline \multicolumn{2}{|l|}{ Age groups, n (\%) } \\
\hline $40-44$ & $170(17.7)$ \\
\hline $45-49$ & $253(26.3)$ \\
\hline $50-54$ & $240(24.9)$ \\
\hline $55-60$ & $299(31.1)$ \\
\hline \multicolumn{2}{|l|}{ Health status, n (\%) } \\
\hline good/very good & $620(64.5)$ \\
\hline fair & $280(29.1)$ \\
\hline poor/very poor & $61(6.3)$ \\
\hline \multicolumn{2}{|l|}{ BMI $\left(k g / m^{2}\right), n(\%)$} \\
\hline$<25$ & $370(40.4)$ \\
\hline $25-29$ & $352(38.5)$ \\
\hline+30 & $193(21.1)$ \\
\hline \multicolumn{2}{|l|}{ Physical activity ${ }^{a}, n(\%)$} \\
\hline low & $284(29.5)$ \\
\hline high & $678(70.5)$ \\
\hline \multicolumn{2}{|l|}{ Household income, n (\%) } \\
\hline low $(<80,000$ USD) & $286(33.0)$ \\
\hline medium & $334(38.5)$ \\
\hline high (> 130,000 USD) & $247(28.5)$ \\
\hline \multicolumn{2}{|l|}{ Educational attainment, n(\%) } \\
\hline low (<12 years schooling) & $213(22.3)$ \\
\hline medium & $645(67.5)$ \\
\hline high (university degree) & $97(10.2)$ \\
\hline \multicolumn{2}{|l|}{ In work force, n(\%) } \\
\hline No & $115(12.0)$ \\
\hline Yes & 847 (88.0) \\
\hline
\end{tabular}

aphysical activity categorized as High: 'daily' or 'several times a week'; Low: 'never or 'once a week or less'

This was also true for those with lower levels of physical activity, with higher BMI and lower self-reported health status. Further, household income and self-reported level of present physical activity were negatively associated with the statement that it was too expensive to go to a fitness center (Table 3) while BMI was positively associated with perceiving cost of exercise as a barrier to maintaining lifestyle changes.

\section{Barriers to life style change: low fat diet}

Some $30 \%$ of respondents reported at least one of the three predefined barriers to dietary change. Among these 


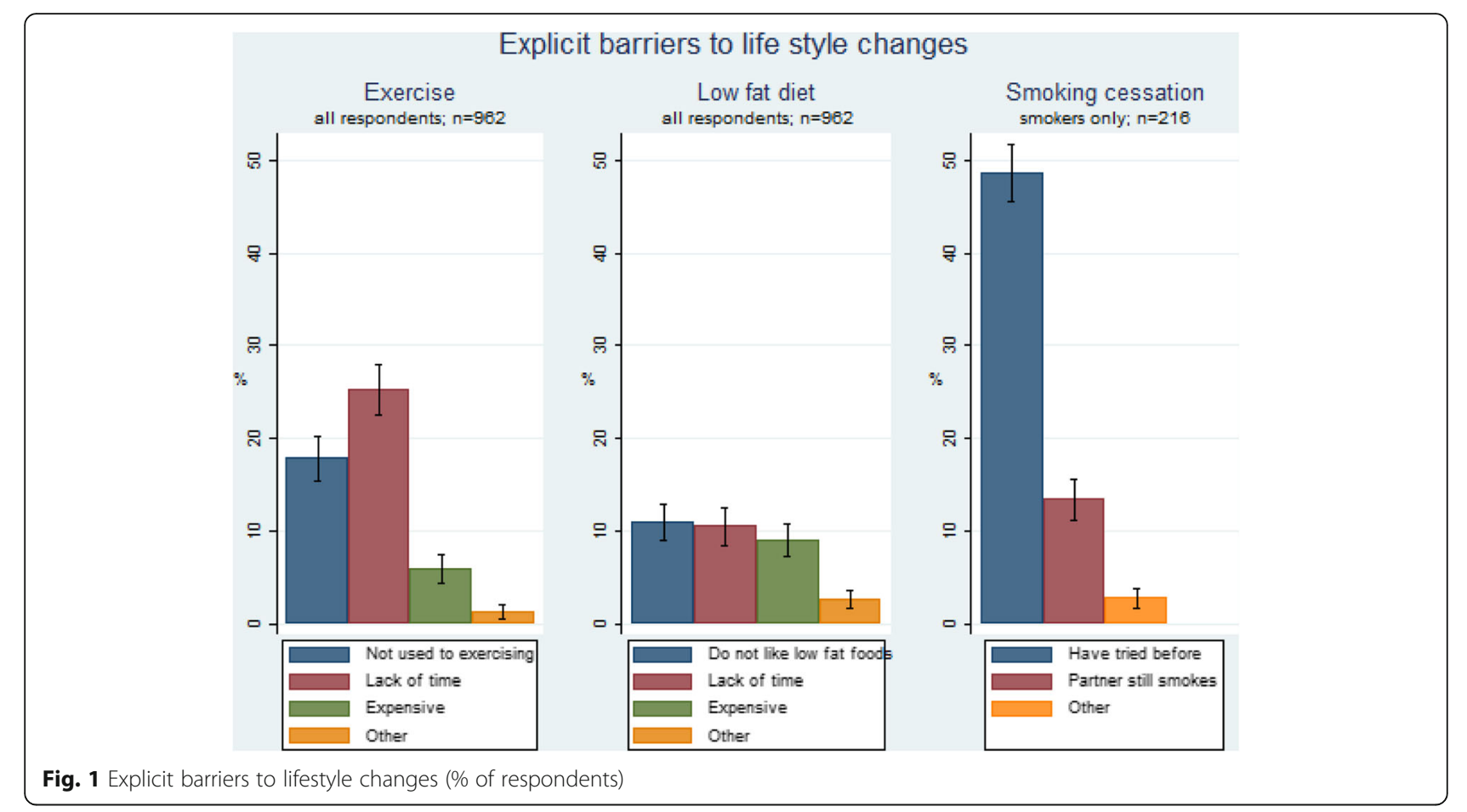

respondents a similar proportion stated barriers relating to not liking low fat food, low fat food taking longer to prepare, and low fat food being too expensive (Fig. 1). Males and individuals with lower self-reported health were more prone to stating that dislike of low fat food was a barrier to lifestyle change (Table 4). Further, the higher the BMI the higher the proportion of respondents reporting increased time demand as a barrier (Table 4).

We also observed that being out of the workforce and high BMI were predictors of reporting monetary costs as a barrier to dietary change.

\section{Barriers to life-style change: smoking cessation}

Among the 216 smokers, at least one of the two predefined barriers to smoking cessation was reported as a barrier to the required lifestyle change by $62 \%$, most of who stated that they had previously unsuccessfully tried to stop smoking (49\%), whereas 'partner still smokes' was stated as a barrier by $13 \%$ of the smokers. No significant associations were found between any of the covariates and the two barriers related to smoking cessation (Table 5).

\section{Barriers to life-style change: free text option}

Analysis of the free text option in the questionnaire did not reveal any major barriers not covered by those themes already included.

\section{Discussion}

The present study indicates that the part of the population at increased risk for CVD (either BMI > 25, or those physically inactive) more often report the specified barriers to lifestyle change than population segments at lower risk for CVD. Even though creating time for physical activity/ exercise or ensuring room in the budget to buy healthier food may be seen as a prioritization issue, our study clearly demonstrates that structural factors, such as low income and being out of the work force are associated with stating that low fat foods are too expensive and that it is too costly to use fitness centers to support lifestyle changes. Similarly, previous studies have shown that being unemployed or having low income and low socioeconomic position is associated with lower participation in and higher drop-out from cardiac rehabilitation programs $[17,18]$. Thus, social inequality exists not only in the delivery and offering of health care services, but also in the ability to take appropriate preventive measures through lifestyle changes to stay healthy. This finding underlines the challenge of social inequality even in an otherwise fairly homogeneous Danish population with apparently equal and cost-free access to health care.

Among the barriers most commonly reported were those relating to cost and time. This may raise the question as to whether some respondents might claim these as barriers because they are aware that they have a health issue needing personal investment (time, money, convenience), but doubt their own ability or true intention to act on it [16]. This could be a kind of coping mechanism transforming the challenge of lifestyle change from being a personal issue to a more societal or structural issue related to costs and lack of time. Testing 
Table 3 Associations between specific barriers for physical exercise and covariates among respondents who indicated a preference for lifestyle changes

\begin{tabular}{|c|c|c|c|c|c|}
\hline & & $\begin{array}{l}\text { Total sample } \\
\text { N }\end{array}$ & $\begin{array}{l}\text { Not used to exercising } \\
\mathrm{n}(\%)^{\mathrm{a}}\end{array}$ & $\begin{array}{l}\text { Lack of time for exercising } \\
\mathrm{n}(\%)^{\mathrm{a}}\end{array}$ & $\begin{array}{l}\text { Too expensive to exercise } \\
\mathrm{n}(\%)^{\mathrm{a}}\end{array}$ \\
\hline \multicolumn{2}{|l|}{ Total } & 962 & $172(17.9)$ & $243(25.3)$ & $57(5.9)$ \\
\hline \multirow[t]{2}{*}{ Gender } & Male & 482 & $93(19.3) p=0.309$ & $134(27.8) p=0.045$ & $29(6.0) p=0.993$ \\
\hline & Female & 480 & 79 (16.5) & $109(22.7)$ & $28(5.8)$ \\
\hline \multirow[t]{4}{*}{ Age group } & $40-44$ & 170 & $22(12.9) p=0.075^{*}$ & $61(35.9) p<0.001^{*}$ & $8(4.7) p=0.144$ \\
\hline & $45-49$ & 253 & $49(19.4)$ & $73(28.9)$ & $18(7.1)$ \\
\hline & $50-54$ & 240 & 37 (15.4) & $58(24.2)$ & $8(3.3)$ \\
\hline & $55-60$ & 299 & $64(21.4)$ & $51(17.1)$ & $23(7.7)$ \\
\hline \multirow[t]{3}{*}{ Health status } & Good/very good & 620 & $78(12.6) p<0.001^{*}$ & $162(26.1) p=0.251$ & $30(4.8) p=0.129$ \\
\hline & Fair & 280 & $75(26.8)$ & $71(25.4)$ & $23(8.2)$ \\
\hline & Poor/very poor & 61 & $19(31.1)$ & $10(16.4)$ & $4(6.6)$ \\
\hline \multirow[t]{3}{*}{ BMl } & $<25$ & 370 & $47(12.7) p<0.001^{*}$ & $100(27.0) p=0.611$ & $9(2.4) p<0.001^{*}$ \\
\hline & $25-29$ & 352 & $60(17.0)$ & $91(25.9)$ & $28(8.0)$ \\
\hline & +30 & 193 & $55(28.5)$ & $47(24.4)$ & $20(10.4)$ \\
\hline \multirow[t]{2}{*}{ Physical activity } & Low & 284 & $126(44.4) p<0.001$ & $98(34.5) p<0.001$ & $27(9.5) p=0.004$ \\
\hline & High & 678 & $46(6.8)$ & $145(21.4)$ & $30(4.4)$ \\
\hline \multirow[t]{3}{*}{ Household income } & Low & 286 & $70(24.5) p=0.001^{*}$ & $54(18.9) p=0.001^{*}$ & $28(9.8) p=0.001^{*}$ \\
\hline & Medium & 334 & $58(17.4)$ & $87(26.0)$ & $13(3.9)$ \\
\hline & High & 247 & $31(12.6)$ & $80(32.4)$ & $8(3.2)$ \\
\hline \multirow[t]{3}{*}{ Educational attainment } & Low & 213 & $45(21.1) p=0.026^{*}$ & $40(18.8) p<0.001^{*}$ & $13(6.1) p=0.159^{*}$ \\
\hline & Medium & 645 & $117(18.1)$ & $160(24.8)$ & $42(6.5)$ \\
\hline & High & 97 & $10(10.3)$ & $42(43.3)$ & $1(1.0)$ \\
\hline \multirow[t]{2}{*}{ In work force } & No & 115 & $28(24.3) p=0.066$ & $7(6.1) p<0.001$ & $12(10.4) p=0.050$ \\
\hline & Yes & 847 & $144(17.0)$ & $236(27.9)$ & $45(5.3)$ \\
\hline
\end{tabular}

All $\mathrm{p}$-values are from multiple logistic regression analyses adjusted for gender and age

- $\mathrm{p}$-value for trend

${ }^{a}$ Numbers $(n)$ and percentages (\%) correspond to the number of patients and proportions (in percent) of patients in the row-category who experienced the given barrier. Note that not all participants experienced a barrier within the theme, and that participants were allowed to select more than one barrier within the theme

subgroup differences in perception of specified physical activity barriers, which had been identified as relevant in prior studies, may unintendedly have prompted some respondents to think only of fitness centers rather than of incorporating 30 min physical activity as a part of everyday life (walking or cycling to work) or without incurring much additional cost by jogging/running in the neighborhood).

Besides structural barriers, we considered more personal barriers related to habits and preferences. Not being used to exercise/physical activity, not liking low fat food, or for smokers having a partner who smokes were each regarded as a barrier to maintaining lifestyle change by at least $10 \%$ of the respondents. This is in accordance with the literature reporting that attitude towards and experience with physical activity/exercise, unwillingness to change diet, and family support are important determinants for maintaining initiated lifestyle changes $[14,17]$.

When perceived barriers are not equally distributed across socio-demographic strata, it underpins the need for a targeted or an individualized approach to CVD prevention through lifestyle changes. Further, it opens for a discussion on the dilemma between a societal wish to promote healthy lifestyles and the existence of population groups unwilling or unable to engage in smoking cessation or in losing weight despite being overweight or obese. In that way, our observations align with previous findings of a discrepancy between what people intend to do if at risk, and what individuals at risk actually do to prevent CVD.

The single most frequently reported barrier to lifestyle changes, stated by $25 \%$ of our respondents, was finding $1 / 2$ an hour extra time to do daily exercise. Demographically these respondents were younger, in the work force, had higher household income and a higher educational attainment. The last three determinants were positively associated with lack of time as perceived barrier for physical exercise, but the associations with age were more complex. Thus, what characterizes this barrier to 
Table 4 Associations between specific barriers for low fat diet and covariates among respondents who indicated a preference for lifestyle changes

\begin{tabular}{|c|c|c|c|c|c|}
\hline & & $\begin{array}{l}\text { Total sample } \\
\mathrm{N}\end{array}$ & $\begin{array}{l}\text { Do not like low fat foods } \\
\mathrm{n}(\%)^{\mathrm{a}}\end{array}$ & $\begin{array}{l}\text { Lack of time for cooking } \\
n(\%)^{\mathrm{a}}\end{array}$ & $\begin{array}{l}\text { Low fat foods are too expensive } \\
\mathrm{n}(\%)^{\mathrm{a}}\end{array}$ \\
\hline \multicolumn{2}{|l|}{ Total } & 962 & $106(11.0)$ & $101(10.5)$ & $87(9.0)$ \\
\hline \multirow[t]{2}{*}{ Gender } & Male & 482 & $83(17.2) p<0.001$ & $54(11.2) p=0.428$ & $47(9.8) p=0.422$ \\
\hline & Female & 480 & $23(4.8)$ & $47(9.8)$ & $40(8.3)$ \\
\hline \multirow[t]{4}{*}{ Age group } & $40-44$ & 170 & $17(10.0) p=0.875^{*}$ & $17(10.0) p=0.366^{*}$ & $18(10.6) p=0.044$ \\
\hline & $45-49$ & 253 & $31(12.3)$ & 30 (11.9) & $30(11.9)$ \\
\hline & $50-54$ & 240 & $25(10.4)$ & $30(12.5)$ & $19(7.9)$ \\
\hline & $55-60$ & 299 & $33(11.0)$ & $24(8.0)$ & $20(6.7)$ \\
\hline \multirow[t]{3}{*}{ Health status } & Good/very good & 620 & $55(8.9) p=0.002^{*}$ & $51(8.2) p=0.008^{*}$ & $48(7.7) p=0.042^{*}$ \\
\hline & Fair & 280 & $38(13.6)$ & $42(15.0)$ & $30(10.7)$ \\
\hline & Poor/very poor & 61 & $13(21.3)$ & $8(13.1)$ & $9(14.8)$ \\
\hline \multirow[t]{3}{*}{ BMl } & $<25$ & 370 & $35(9.5) p=0.034$ & $26(7.0) p=0.001^{*}$ & $17(4.6) p<0.001^{*}$ \\
\hline & $25-29$ & 352 & $37(10.5)$ & $37(10.5)$ & $37(8.0)$ \\
\hline & +30 & 193 & $32(16.6)$ & $32(16.6)$ & $28(14.5)$ \\
\hline \multirow[t]{2}{*}{ Physical activity } & Low & 284 & $34(12.0) p=0.636$ & $32(11.3) p=0.626$ & $21(7.4) p=0.231$ \\
\hline & High & 678 & $72(10.6)$ & 69 (10.2) & $66(9.7)$ \\
\hline \multirow[t]{3}{*}{ Household income } & Low & 286 & $36(12.6) p=0.078^{*}$ & $21(7.3) p=0.154^{*}$ & $35(12.2) p=0.012^{*}$ \\
\hline & Medium & 334 & $42(12.6)$ & $42(12.6)$ & $33(9.9)$ \\
\hline & High & 247 & $21(8.5)$ & $28(11.3)$ & $15(6.1)$ \\
\hline \multirow[t]{3}{*}{ Educational attainment } & Low & 213 & $24(11.3) \mathrm{p}=0.428^{*}$ & $30(14.1) p=0.115^{*}$ & $28(13.1) p=0.005^{*}$ \\
\hline & Medium & 645 & $71(11.0)$ & $63(9.8)$ & $57(8.8)$ \\
\hline & High & 97 & $11(11.3)$ & $8(8.2)$ & $2(2.1)$ \\
\hline \multirow[t]{2}{*}{ In work force } & No & 115 & $10(8.7) p=0.761$ & $10(8.7) p=0.639$ & $19(16.5) p=0.001$ \\
\hline & Yes & 847 & $96(11.3)$ & $91(10.7)$ & $68(8.0)$ \\
\hline
\end{tabular}

All $p$-values are from multiple logistic regression analyses adjusted for gender and age

* $\mathrm{p}$-value for trend

${ }^{a}$ Numbers ( $n$ ) and percentages (\%) correspond to the number of patients and proportions (in percent) of patients in the row-category who experienced the given barrier. Note that not all participants experienced a barrier within the theme, and that participants were allowed to select more than one barrier within the theme

lifestyle change is diversity, which may also be part of the explanation why only some but not all previous studies have found lack of time or time stress to be a barrier to lifestyle changes [6, 14].

Unlike the barriers on diet and physical activity, we found no statistically significant sociodemographic/socioeconomic gradients for perceived barriers to smoking cessation. For educational attainment and health status this may be due to the reduced sample size.

In the present study, the respondents were presented with a limited number of suggested barriers to consider as well as a free text option. This may be seen as a limitation, since it might direct the respondent's attention to the barriers suggested in the questionnaire which might lead to underestimation of the overall number of barriers perceived by people trying to change their lifestyle. However, the purpose of the present study was not to explore the overall number and range of possible barriers, but to test subgroup-specific differences in barriers which had been identified as the most relevant ones in the literature [14, 17, 19]. Further, examining the free text option in the questionnaire did not reveal any major topics not already covered by the themes. Nevertheless it is possible that the specification of only 2 to 3 specific options, because of cognitive availability, acquiescence bias or conformity processes, may have led to higher endorsement of these barriers at the expense of other, nonlisted choices. We do multiple comparisons and therefore have an increased risk for type I errors. We have handled this by focusing on differences that are both relevant in size and having $p$-values below 0.005 . Another important limitation may be that our respondents were asked to state their intentions and beliefs referring to a hypothetical risk situation, rather than to a real-life CVD risk. However, the subsample of our population reporting either to have a heart disease themselves or having family members with heart disease (in total $28 \%$ of our respondents) gave similar responses to barriers 
Table 5 Associations between specific barriers to smoking cessation and covariates among respondents who indicated a preference for lifestyle changes

\begin{tabular}{|c|c|c|c|c|}
\hline & & $\begin{array}{l}\text { Total sample of smokers } \\
\mathrm{N}\end{array}$ & $\begin{array}{l}\text { Have tried before } \\
\text { n (\%) }\end{array}$ & $\begin{array}{l}\text { Partner still smokes } \\
\mathrm{n}(\%)\end{array}$ \\
\hline \multicolumn{2}{|l|}{ Total } & 216 & $105(48.6)$ & $29(13.4)$ \\
\hline \multirow[t]{2}{*}{ Gender } & Male & 115 & $56(48.7) p=0.992$ & $13(11.3) p=0.318$ \\
\hline & Female & 101 & $49(48.5)$ & $16(15.8)$ \\
\hline \multirow[t]{4}{*}{ Age group } & $40-44$ & 47 & $28(59.6) p=0.051$ & $5(10.6) p=0.360$ \\
\hline & $45-49$ & 53 & $26(49.1)$ & $7(13.2)$ \\
\hline & $50-54$ & 53 & $26(49.1)$ & $6(11.3)$ \\
\hline & $55-60$ & 63 & $25(39.7)$ & $11(17.5)$ \\
\hline \multirow[t]{3}{*}{ Health status } & Good/very good & 115 & $60(52.2) p=0.560^{*}$ & $11(9.6) p=0.183^{*}$ \\
\hline & Fair & 83 & $34(41.0)$ & $16(19.3)$ \\
\hline & Poor/very poor & 18 & $11(61.1)$ & $2(11.1)$ \\
\hline \multirow[t]{3}{*}{ BMl } & $<25$ & 89 & $42(47.2) p=0.949$ & $13(14.6) p=0.744^{\prime}$ \\
\hline & $25-29$ & 72 & $38(52.1)$ & $9(12.3)$ \\
\hline & +30 & 45 & $20(44.4)$ & $6(13.3)$ \\
\hline \multirow[t]{2}{*}{ Physical activity } & Low & 84 & $41(48.8) p=0.835$ & $12(14.3) p=0.839$ \\
\hline & High & 132 & $64(48.5)$ & $17(12.9)$ \\
\hline \multirow[t]{3}{*}{ Household income } & Low & 82 & $37(45.1) p=0.892^{*}$ & $12(14.6) p=0.904$ \\
\hline & Medium & 66 & $34(51.5)$ & $8(12.1)$ \\
\hline & High & 44 & $20(45.5)$ & $7(15.9)$ \\
\hline \multirow[t]{3}{*}{ Educational attainment } & Low & 65 & $38(58.5) p=0.154^{*}$ & $9(13.8) p=0.623^{*}$ \\
\hline & Medium & 130 & $58(44.6)$ & $18(13.8)$ \\
\hline & High & 20 & $9(45.0)$ & $2(10.0)$ \\
\hline \multirow[t]{2}{*}{ In work force } & No & 35 & $17(48.6) p=0.732$ & $2(5.7) p=0.105$ \\
\hline & Yes & 181 & 88 (48.6) & $27(14.9)$ \\
\hline
\end{tabular}

All $p$-values are from multiple logistic regression analyses adjusted for gender and age

- p-value for trend

${ }^{a}$ Numbers ( $n$ ) and percentages (\%) correspond to the number of patients and proportions (in percent) of patients in the row-category who experienced the given barrier. Note that not all participants experienced a barrier within the theme, and that participants were allowed to select more than one barrier within the theme

for lifestyle changes as the majority of our respondents with no prior experience with heart disease. As the present study population is extracted from a representative population sample based on criteria on willingness to change lifestyle and avoid medication, we cannot expect the chosen sample to be fully representative of the entire population. However, from previous data from Statistics Denmark we have previously reported that within the present age group the average household income is appr. 100,000 USD, 28\% have less than 12 years schooling, and $7 \%$ have a University degree [20]. Thus, despite that our group is a subpopulation based on the above mentioned criteria; they appear fairly representative of the general population regarding these sociodemographic characteristics. Further strengths of the study were the solid sample size of 962 respondents, the high participation rate (91\%) among those accessing the website for the study, that respondents were not informed about the specific risk scenarios before entering the survey, and that the questionnaire was thoroughly tested before being used.

An important observation from our study is the considerable heterogeneity between different social groups in the population regarding a number of barriers to lifestyle change, an issue, which has so far not been given sufficient attention. Thus, traditional generalized preventive efforts based on 'one size fits all' may not be optimal. A recent review concludes that prevention is costeffective and should be delivered at the general population level by promoting healthy lifestyle behavior, and at the individual level by tackling unhealthy lifestyles (e.g. poor-quality diets, physical inactivity, smoking) [7]. This is in accordance with our study, suggesting to change or supplement traditional general public campaigns to counter CVD by using individualized and targeted initiatives directed at the groups at highest risk. 


\section{Conclusion}

We observed a considerable heterogeneity between different social groups in the population regarding a number of barriers to lifestyle change. Our study demonstrates that social inequality exists in the anticipated ability to take appropriate preventive measures through lifestyle changes to stay healthy. While our study is based on a hypothetical scenario and any conclusions are therefore tentative, the findings suggest that it may be worth wile to consider supplementing traditional public campaigns to counter cardiovascular disease, for instance by using individualized and targeted initiatives directed at the groups at highest risk.

\section{Acknowledgements}

Not applicable.

\section{Funding}

This research received no grant from any funding agency in the public, commercial or not-for-profit sections.

\section{Availability of data and materials}

The datasets used and analysed as part of the current study is available from the corresponding author on reasonable request.

\section{Authors' contributions}

$J B N, D G H$, and PVL contributed to the design of the work. JBN drafted the work. PVL did the statistics. JBN, AL, DGH, DEJ, JS, and PVL all contributed significantly to data analysis and interpretation of data, critically revised the manuscript, and gave final approval before submission.

\section{Ethics approval and consent to participate}

According to the research ethics committee system in Denmark, the present study is a questionnaire study not involving human biological material and therefore fall outside the demarcation of projects, which have to be approved of by a regional research ethics committee (Danish Act on Research Ethics Review of Health Research Projects; $\$ 14$ stk.2, June 2011). When signing in as member of the TNS Gallup web-panel, participants give consent to third parties to use and publish in anonymous form all information collected.

\section{Consent for publication}

When signing in as member of the TNS Gallup web-panel, participants give consent to third parties to use and publish in anonymous form all information collected.

\section{Competing interests}

The authors declare that they have no competing interests.

\section{Publisher's Note}

Springer Nature remains neutral with regard to jurisdictional claims in published maps and institutional affiliations.

\section{Author details}

${ }^{1}$ Research Unit for General Practice, Department of Public Health, University of Southern Denmark, J.B.Winsløwvej 9, DK-5000 Odense, Denmark. ${ }^{2}$ Unit for Health Promotion Research, Department of Public Health, University of Southern Denmark, Niels Bohrs Vej 9, DK-6700 Esbjerg, Denmark. ${ }^{3}$ COHERE, Department of Public Health, University of Southern Denmark, J.B.Winsløwvej 9, DK-5000 Odense, Denmark. ${ }^{4}$ Epidemiology, Biostatistics and Biodemography, Department of Public Health, University of Southern Denmark, J.B.Winsløwvej 9, DK-5000 Odense, Denmark.

Received: 22 December 2016 Accepted: 4 September 2017 Published online: 12 September 2017

\section{References}

1. Juel K. Dødeligheden i Danmark gennem 100 år: Danskerne lever længere, men hvorfor 3-4 år kortere end svenske mænd og franske kvinder?
Copenhagen: National Institute for Public Health; 2004. (report in DanishMortality in Denmark through 100 years)

2. Moran AE, Forouzanfar MH, Roth GA, Mensah GA, Ezzati M, Murray CJ, et al. Temporal trends in ischemic heart disease mortality in 21 world regions, 1980 to 2010: the global burden of disease 2010 study. Circulation. 2014;129:1483-92.

3. Liu K, Daviglus ML, Loria CM, Colangelo LA, Spring B, Moller AC, et al. Healthy lifestyle through young adulthood and the presence of low cardiovascular disease risk profile in middle age: the coronary artery risk development in (young) adults (CARDIA) study. Circulation. 2012;125:996-1004.

4. Larsson SC, Tektonidis TG, Gigante B, Åkesson A, Wolk A. Healthy lifestyle and risk of heart failure - results from 2 prospective cohort studies. Circ Heart Failure. 2016;9:e002855.

5. Jorgensen T, Jacobsen RK, Toft U, Aadahl M, Glümer C, Pisinger C. Effect of screening and lifestyle counselling on incidence of ischaemic heart disease in general population: Inter99 randomised trial. BMJ. 2014;348:93617.

6. Kotseva K, Wood D, De Bacquer D, De Backer G, Rydén L, Jennings C, et al. EUROASPIRE IV: a European society of cardiology survey on the lifestyle, risk factor and therapeutic management of coronary patients from 24 European countries. Eur J Prev Cardiol. 2016;23:636-48.

7. Piepoli MF, Hoes AW, Agewall S, Albus C, Brotons C, Catapano AL, et al. 2016 European guidelines on cardiovascular disease prevention in clinical practice: the sixth joint task force of the European society of cardiology and other societies on cardiovascular disease prevention in clinical practice (constituted by representatives of 10 societies and by invited experts): developed with the special contribution of the European association for cardiovascular prevention \& rehabilitation (EACPR). Eur Heart J. 2016;37:2315-81.

8. Costello E, Kafchinski M, Vrazel JE, Sullivan P. Motivators, barriers, and beliefs regarding physical activity in an older adult population. J Geriat Phys Therap. 2011;34:138-47.

9. Lara J, McCrum LA, Mathers JC. Association of mediterranean diet and other health behaviours with barriers to healthy eating and perceived health among British adults of retirement age. Maturitas. 2014;79:292-8.

10. Mailey EL, Huberty J, Dinkel D, McAuley E. Physical activity barriers and facilitators among working mothers and fathers. BMC Public Health. 2014;14:657.

11. Osuji T, Lovegreen SL, Elliott M, Brownson RC. Barriers to physical activity among women in the rural midwest. Women Health. 2006;44:41-55.

12. Petter M, Blanchard C, Kemp KAR, Mazoff AS, Ferrier SN. Correlates of exercise among coronary heart disease patients: review, implications and future directions. Eur J Cardiovasc Prev Rehabil. 2009;16:515-26.

13. Twyman L, Bonevski B, Paul C, Bryant J. Perceived barriers to smoking cessation in selected vulnerable groups: a systematic review of the qualitative and quantitative literature. BMJ Open. 2014;4:e006414.

14. Murray J, Honey S, Hill K, Craigs C, House A. Individual influences on lifestyle change to reduce vascular risk: a qualitative literature review. $\mathrm{Br} J \mathrm{Gen}$ Practice. 2012;62:e403-10.

15. Nielsen JB, Jarbøl DE, Gyrd-Hansen D, Barfoed BML, Larsen PV. Determinants for acceptance of preventive treatment against heart disease - a web-based population survey. BMC Public Health. 2014;14:783-91.

16. Jarbøl DE, Larsen PV, Gyrd-Hansen D, Søndergaard J, Brandt C, et al. Determinants for preferences for lifestyle changes or medication and beliefs in ability to retain lifestyle changes. A population-based survey. Prev Med Rep. 2017;6:66-73.

17. Worcester MU, Murphy BM, Mee VK, Roberts SB, Goble AJ. Cardiac rehabilitation programmes: predictors of non-attendance and drop-out. Eur J Cardiovasc Prev Rehabil. 2004;11:328-35.

18. Nielsen KM, Faergeman O, Foldspang A, Larsen ML. Cardiac rehabilitation: health characteristics and socio-economic status among those who do not attend. Eur J Pub Health. 2008;18:479-83.

19. Murray J, Craigs CL, Hill KM, Honey S, House A. A systematic review of patient reported factors associated with uptake and completion of cardiovascular lifestyle behavior change. BMC Cardiovasc Disord. 2012;12:120-32.

20. Støvring H, Gyrd-Hansen D, Kristiansen IS, Nexøe J, Nielsen JB. Communicating effectiveness of intervention for chronic diseases: what single format can replace comprehensive information? BMC Med Inform Decis Mak. 2008;8:25-33. 